\title{
MULTI-WAVELENGTH PROPERTIES OF THE TYPE IIb SN 2008ax
}

\author{
P. W. A. Roming ${ }^{1}$, T. A. Pritchard ${ }^{1}$, P. J. Brown ${ }^{1}$, S. T. Holland ${ }^{2,3}$, S. Immler ${ }^{4,5}$, C. J. Stockdale ${ }^{6}$, K. W. Weiler ${ }^{7}$, \\ N. Panagia ${ }^{8,9,10}$, S. D. Van DyK ${ }^{11}$, E. A. Hoversten ${ }^{1}$, P. A. Milne ${ }^{12}$, S. R. OAtes ${ }^{13}$, B. Russell ${ }^{14}$, And C. VAndrevala ${ }^{6}$ \\ ${ }^{1}$ Department of Astronomy \& Astrophysics, Penn State University, 525 Davey Laboratory, University Park, PA 16802, USA; roming@ astro.psu.edu \\ ${ }^{2}$ Universities Space Research Association, 10227 Wincopin Circle, Suite 500, Columbia, MD 21044, USA \\ ${ }^{3}$ Center for Research \& Exploration in Space Science \& Technology, Code 668.8, Greenbelt, MD 20771, USA \\ ${ }^{4}$ NASA/Goddard Space Flight Center, Astrophysics Science Division, Code 662, Greenbelt, MD 20771, USA \\ ${ }^{5}$ Department of Astronomy, University of Maryland, College Park, MD 20742, USA \\ ${ }^{6}$ Physics Department, Marquette University, P.O. Box 1881, Milwaukee, WI 53201-1881, USA \\ ${ }^{7}$ Naval Research Laboratory, Code 7210, Washington, DC 20375-5320, USA \\ ${ }^{8}$ Space Telescope Science Institute, 3700 San Martin Dr, Baltimore, MD 21218, USA \\ ${ }^{9}$ INAF-CT Osservatorio Astrofisico di Catania, Via S. Sofia 79, I-95123 Catania, Italy \\ ${ }^{10}$ Supernova Ltd, OYV \#131, Northsound Rd, Virgin Gorda, British Virgin Islands \\ ${ }^{11}$ Spitzer Science Center, IPAC, California Institute of Technology, M/C 220-6, Pasadena, CA 91125, USA \\ ${ }^{12}$ Department of Astronomy, University of Arizona, Tucson, AZ 85721, USA \\ ${ }^{13}$ Mullard Space Science Laboratory, University College London, Holmbury St. Mary, Dorking, Surrey RH5 6NT, UK \\ ${ }^{14}$ Department of Physics, University of Maryland, College Park, MD 20742, USA \\ Received 2009 August 31; accepted 2009 September 17; published 2009 October 5
}

\begin{abstract}
We present the UV, optical, X-ray, and radio properties of the Type IIb SN 2008ax discovered in NGC 4490. The observations in the UV are one of the earliest of a Type Ilb supernova (SN). On approximately day 4 after the explosion, a dramatic upturn in the $u$ and $u v w 1\left(\lambda_{c}=2600 \AA\right)$ light curves occurred after an initial rapid decline which is attributed to adiabatic cooling after the initial shock breakout. This rapid decline and upturn is reminiscent of the Type IIb SN 1993J on day 6 after the explosion. Optical/near-IR spectra taken around the peak reveal prominent $\mathrm{H} \alpha, \mathrm{He}$, and Ca II absorption lines. A fading X-ray source is also located at the position of SN 2008ax, implying an interaction of the SN shock with the surrounding circumstellar material and a mass-loss rate of the progenitor of $\dot{M}=(9 \pm 3) \times 10^{-6} M_{\odot} \mathrm{yr}^{-1}$. The unusual time evolution (14 days) of the $6 \mathrm{~cm}$ peak radio luminosity provides further evidence that the mass-loss rate is low. Combining the UV, optical, X-ray, and radio data with models of helium exploding stars implies the progenitor of SN 2008ax was an unmixed star in an interacting binary. Modeling of the SN light curve suggests a kinetic energy $\left(E_{k}\right)$ of $0.5 \times 10^{51} \mathrm{erg}$, an ejecta mass $\left(M_{\mathrm{ej}}\right)$ of $2.9 M_{\odot}$, and a nickel mass $\left(M_{\mathrm{Ni}}\right)$ of $0.06 M_{\odot}$.
\end{abstract}

Key words: radio continuum: stars - supernovae: individual (SN 2008ax) - ultraviolet: stars - X-rays: stars

\section{INTRODUCTION}

Type IIb supernovae (SNe), first proposed by Woosley et al. (1987), were suggested to be the result of the core collapse of a Type $\mathrm{Ib}$ progenitor that has a small, but non-negligible $\left(\sim 10^{-1} M_{\odot}\right.$; Pastorello et al. 2008 , hereafter P08), H-envelope. This SN type is arguably one of the rarest and most interesting. Only $\sim 40$ have been discovered in the last $20+$ years and only a handful, such as SNe 1987K (Filippenko 1988), 1993J (Nomoto et al. 1993), 1996cb (Qiu et al. 1999), and 2008ax (P08), have been well observed.

In this Letter, we report on imaging observations of SN 2008ax with the Swift (Gehrels et al. 2004) Ultra-Violet/Optical Telescope (UVOT; Roming et al. 2005) and X-Ray Telescope (XRT; Burrows et al. 2005), the Chandra Advanced CCD Imaging Spectrometer (ACIS), and the Very Large Array (VLA), as well as spectroscopic observations with the Hobby-Eberly Telescope (HET; Ramsey et al. 1998). The UVOT observations are one of the earliest reported observations in the UV of a Type IIb $\mathrm{SNe}$, second only to the International Ultraviolet Explorer (IUE) observations of SN 1993J (de Boer et al. 1993).

\section{OBSERVATIONS AND DATA REDUCTIONS}

SN 2008ax (Figure 1) was discovered on 2008 March 3.45 (UT) in NGC 4490 (with LMC-like metallicity) at R.A.J2000 $=$ $12^{\mathrm{h}} 30^{\mathrm{m}} 40^{\mathrm{s}} .80$, decl. ${ }_{\text {J2000 }}=+41^{\circ} 38^{\prime} 14^{\prime \prime} .5$ (Mostardi et al. 2008).
Based on this detection time and the non-detection of the SN approximately $6 \mathrm{hr}$ earlier, P08 set the time of the shock breakout at $\mathrm{JD}=2454528.80 \pm 0.15$, which is adopted here.

The UVOT observed SN 2008ax from 2008 March 4 $(\mathrm{JD}=2454530.16)$ to 2008 April $26(\mathrm{JD}=2454582.52)$. Observations were performed with a cadence varying between 2 and 6 days using three optical $(u, b, v)$ and three UV (uvw2, uvm 2, uvw1: $\lambda_{c}=1928,2246,2600 \AA$, respectively; Poole et al. 2008) filters. A later observation on 2008 November 23 was made after the SN had faded for use as a galaxy subtraction template. Photometry using a 3 " source aperture, including template galaxy flux subtraction, was performed following the method outlined in Brown et al. (2009). The data reduction pipeline used the HEASOFT 6.6.3 and Swift Release 3.3 analysis tools with UVOT zero points from Poole et al. (2008). The resulting light curves are presented in Table 1 and Figure 2.

We note that for the majority of SNe analyzed with Swift analysis tools, UVOT photometry is well behaved. However, in the case of SN 2008ax, the exceptionally bright host galaxy underlying the SN leads to small offsets in magnitude due to coincidence losses (see Fordham et al. 2000) in bright extended backgrounds. This results in the reported magnitudes being $0.23 \pm 0.07$ ( $b$ filter) and $0.19 \pm 0.06$ ( $v$ filter) fainter than the P08 magnitudes. Although this offset influences the UVOT $u$ band as well, the $u$ filter has more throughput in the blue than ground-based $U$ filters. This is the most probable contributor to the $\sim 0.6$ mag increase in the $u$-band peak as compared to P08. 

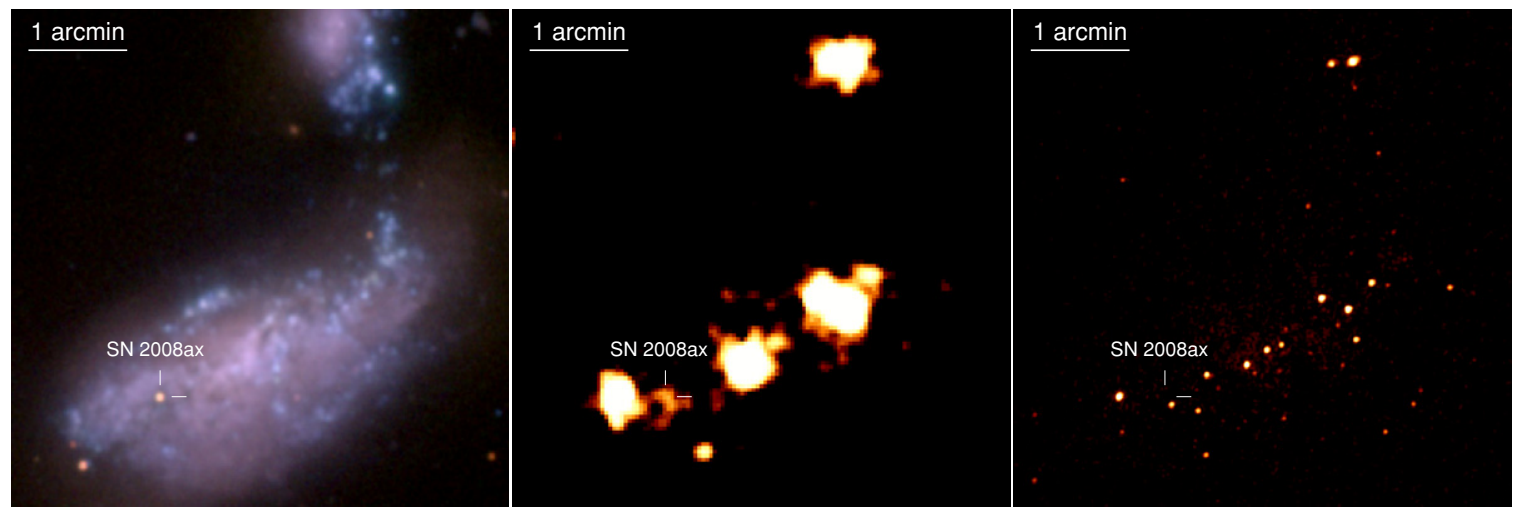

Figure 1. Swift UVOT (left), Swift XRT (middle), and Chandra (right) images of SN 2008ax and its host galaxy. Left: the UV/optical image was constructed from the UVOT $b(284 \mathrm{~s}$; red), $u$ (285 s; green), and $u v w 1$ (570 s; blue) filters obtained on 2008 March 4. Positions are indicated by white tick marks. Middle: X-ray image $(0.2-10 \mathrm{keV})$ from the merged $51.3 \mathrm{ks}$ XRT data. The image is smoothed with a Gaussian filter of 3 pixels (7". 1) FWHM. Right: X-ray image (0.2-10 keV) constructed from the 97.6 ks ACIS-S pre-explosion data smoothed with a Gaussian filter of 3 pixels (1".5) FWHM.

Table 1

SN 2008ax UV/Optical Characteristics

\begin{tabular}{|c|c|c|c|c|c|c|}
\hline \multirow{2}{*}{$\begin{array}{c}\text { Time } \\
(\mathrm{JD} 2454500+)\end{array}$} & \multicolumn{6}{|c|}{ Observed Magnitudes } \\
\hline & $u v w 2$ & uvm 2 & $u v w 1$ & $u$ & $b$ & $v$ \\
\hline $30.5 \pm 0.3$ & (19.93) & $\ldots$ & $18.95 \pm 0.30$ & $17.21 \pm 0.10$ & $17.61 \pm 0.13$ & $\ldots$ \\
\hline $32.9 \pm 0.1$ & $\ldots$ & $\ldots$ & (19.67) & $17.96 \pm 0.16$ & $17.10 \pm 0.08$ & $15.97 \pm 0.07$ \\
\hline $36.1 \pm 0.0$ & $\ldots$ & $\ldots$ & $(17.37)$ & $\ldots$ & $\ldots$ & $\ldots$ \\
\hline $38.4 \pm 0.1$ & $\ldots$ & $\ldots$ & $17.08 \pm 0.09$ & $15.22 \pm 0.05$ & $15.25 \pm 0.05$ & $14.56 \pm 0.05$ \\
\hline $40.2 \pm 0.1$ & $17.98 \pm 0.16$ & $(19.17)$ & $16.61 \pm 0.09$ & $14.69 \pm 0.05$ & $14.88 \pm 0.05$ & $14.27 \pm 0.05$ \\
\hline $44.1 \pm 0.4$ & $17.61 \pm 0.10$ & $19.20 \pm 0.40$ & $15.98 \pm 0.06$ & $14.17 \pm 0.05$ & $14.49 \pm 0.05$ & $13.91 \pm 0.04$ \\
\hline $46.6 \pm 0.1$ & $17.51 \pm 0.11$ & $18.64 \pm 0.34$ & $15.94 \pm 0.06$ & $14.06 \pm 0.04$ & $14.32 \pm 0.04$ & $13.72 \pm 0.04$ \\
\hline $50.1 \pm 0.0$ & $17.84 \pm 0.20$ & $\ldots$ & $16.45 \pm 0.08$ & $14.52 \pm 0.05$ & $14.38 \pm 0.04$ & $\ldots$ \\
\hline $53.8 \pm 0.2$ & $18.50 \pm 0.20$ & $\ldots$ & $17.10 \pm 0.10$ & $15.23 \pm 0.05$ & $14.66 \pm 0.05$ & $13.76 \pm 0.04$ \\
\hline $54.6 \pm 1.0$ & $\ldots$ & (19.42) & $\ldots$ & $\ldots$ & $\ldots$ & $\ldots$ \\
\hline $55.6 \pm 0.0$ & $18.80 \pm 0.29$ & $\ldots$ & $17.57 \pm 0.16$ & $15.82 \pm 0.06$ & $15.04 \pm 0.05$ & $14.02 \pm 0.04$ \\
\hline $61.2 \pm 0.6$ & $\ldots$ & $\ldots$ & $18.88 \pm 0.41$ & $17.06 \pm 0.11$ & $15.75 \pm 0.05$ & $14.41 \pm 0.05$ \\
\hline $62.4 \pm 1.8$ & (19.85) & (19.57) & $\ldots$ & $\ldots$ & $\ldots$ & $\ldots$ \\
\hline $64.1 \pm 0.1$ & $\ldots$ & $\ldots$ & $18.46 \pm 0.25$ & $17.32 \pm 0.12$ & $15.99 \pm 0.05$ & $14.67 \pm 0.05$ \\
\hline $67.8 \pm 0.1$ & $\ldots$ & $\ldots$ & $19.16 \pm 0.37$ & $17.49 \pm 0.12$ & $16.24 \pm 0.06$ & $14.84 \pm 0.05$ \\
\hline $72.0 \pm 0.2$ & $\ldots$ & $\ldots$ & $19.52 \pm 0.51$ & $17.65 \pm 0.13$ & $16.43 \pm 0.06$ & $14.97 \pm 0.07$ \\
\hline $74.0 \pm 0.1$ & $\cdots$ & $\cdots$ & $19.37 \pm 0.44$ & $17.68 \pm 0.13$ & $16.50 \pm 0.06$ & $15.07 \pm 0.06$ \\
\hline $75.5 \pm 0.0$ & $\ldots$ & $\ldots$ & $19.24 \pm 0.39$ & $17.62 \pm 0.13$ & $16.50 \pm 0.06$ & $15.09 \pm 0.05$ \\
\hline $78.8 \pm 0.1$ & $19.64 \pm 0.45$ & $\ldots$ & $19.44 \pm 0.48$ & $17.67 \pm 0.13$ & $16.54 \pm 0.06$ & $15.22 \pm 0.05$ \\
\hline $81.0 \pm 2.3$ & $\ldots$ & (19.84) & $\ldots$ & $\ldots$ & $\ldots$ & $\ldots$ \\
\hline $82.2 \pm 0.2$ & $\ldots$ & $\ldots$ & $19.37 \pm 0.47$ & $17.59 \pm 0.13$ & $16.56 \pm 0.06$ & $15.27 \pm 0.05$ \\
\hline $82.6 \pm 0.7$ & (19.92) & $\ldots$ & $\ldots$ & $\ldots$ & $\ldots$ & $\ldots$ \\
\hline $75.5 \pm 0.4$ & $\ldots$ & $\cdots$ & $19.53 \pm 0.53$ & $17.61 \pm 0.13$ & $16.68 \pm 0.07$ & $15.29 \pm 0.05$ \\
\hline$m_{\text {Peak }}(\mathrm{mag})$ & $17.47 \pm 0.09$ & $18.46 \pm 0.35$ & $15.90 \pm 0.05$ & $14.05 \pm 0.04$ & $14.30 \pm 0.04$ & $13.56 \pm 0.06$ \\
\hline$M_{\text {Peak }}(\mathrm{mag})$ & $-12.45 \pm 0.31$ & $-11.21 \pm 0.45$ & $-14.02 \pm 0.29$ & $-15.87 \pm 0.29$ & $-15.62 \pm 0.29$ & $-16.36 \pm 0.30$ \\
\hline$t_{\text {Peak }}(\mathrm{JD} 2454500+)$ & $46.3 \pm 1.4$ & $49.1 \pm 5.3$ & $45.6 \pm 0.5$ & $46.1 \pm 0.4$ & $47.6 \pm 0.8$ & $50.4 \pm 0.7$ \\
\hline$t_{\text {Rise }}$ (days) & $17.5 \pm 1.4$ & $20.2 \pm 5.3$ & $16.8 \pm 0.6$ & $17.2 \pm 0.4$ & $18.8 \pm 0.8$ & $21.8 \pm 0.7$ \\
\hline
\end{tabular}

Note. ${ }^{a}$ Magnitudes in parenthesis are upper limit.

Since the background galaxy count rate is significantly lower in the UV, the UV filters are not affected by this undersampling.

To determine the peak magnitude $\left(m_{\text {Peak }}\right)$ and time of peak $\left(t_{\text {Peak }}\right)$ in each filter, $10^{5}$ Monte Carlo simulations fitting a cubic spline to the data points in each filter were performed. The mean of the distributions were taken as the peak times and magnitudes, with the standard deviations as the errors (Table 1). Rise times to peak $\left(t_{\text {Rise }}\right)$ and absolute magnitudes $\left(M_{\text {Peak }}\right)$ were calculated using the time of shock breakout as the temporal zero point and the distance modulus ( $\mu=29.92 \pm 0.29 \mathrm{mag})$, respectively, as determined in P08. Errors were calculated in quadrature (Table 1). None of the magnitudes include extinction or $k$-corrections. Since $u v m 2$ filter observations only provide two detections, the upper limits were treated as detections for the simulations; therefore, $t_{\text {Peak }}$ and $t_{\text {Rise }}$ are probably lower than reported.

To calculate $M_{\text {Peak }}$, a spectrum of a similar Type IIb SN (1993J; Jeffery et al. 1994) taken near peak was de-reddened and redshifted to the rest frame for use as a template. Milky Way (MW) and host extinction were then computed using the $E(B-V)$ value from P08 and applied to our spectral template using the Cardelli et al. (1989) MW and Pei (1992) 

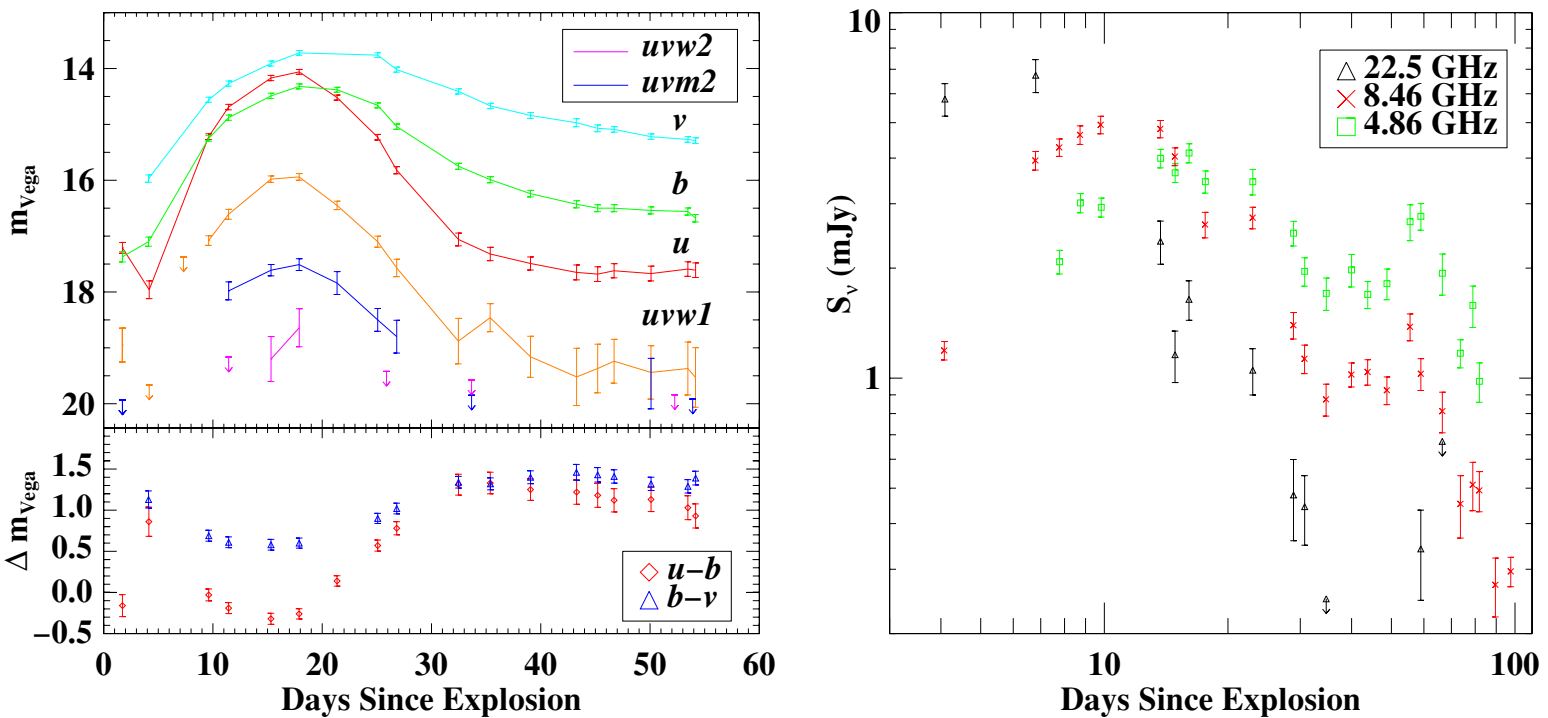

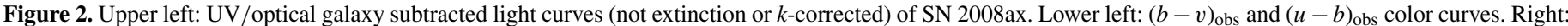
radio light curves. Phases are computed since explosion.

Table 2

Extinction and\& $k$-Correction Factors

\begin{tabular}{lcccccc}
\hline \hline UVOT Filter & $u v w 2$ & $u v m 2$ & $u v w 1$ & $u$ & $b$ & $v$ \\
\hline Extinction (host + MW) & -2.05 & -2.44 & -1.74 & -1.47 & -1.24 & -0.95 \\
$k$-Correction & -0.06 & -0.09 & -0.09 & -0.10 & -0.02 & +0.01
\end{tabular}

LMC laws, respectively. Extinction and $k$-corrections (Table 2) were computed using spectrophotometric methods; extinction corrections were computed via the subtraction of synthetic magnitudes of an unreddened and reddened template spectrum in the observed frame, while $k$-corrections were computed via the subtraction of synthetic magnitudes from the unreddened template spectrum in the rest and observed frames.

We analyzed all XRT observations obtained between 2009 March 5 and 2009 March 26. Due to a nearby X-ray source (7" from the SN), X-ray counts were extracted within a 2-pixel (4".8) radius circular region centered on the optical position of the SN. The background was extracted locally to account for detector and sky background, and unresolved emission from the host. An

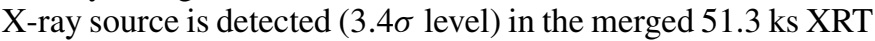
data (Figure 1, middle) at the position of the SN with a pointspread function (PSF), sampling dead time, and vignettingcorrected net count rate of $(6.4 \pm 1.9) \times 10^{-4}$ counts s $^{-1}$. Adopting a thermal plasma spectrum $(k T=10 \mathrm{keV}$; see Fransson et al. 1996) and assuming a Galactic foreground column density with no intrinsic absorption $\left(N_{\mathrm{H}}=1.78 \times 10^{20} \mathrm{~cm}^{-2}\right.$; Dickey \& Lockman 1990) gives a $0.2-10 \mathrm{keV}$ flux and luminosity of $f_{0.2-10}=(3.0 \pm 0.9) \times 10^{-14} \mathrm{erg} \mathrm{cm}^{-2} \mathrm{~s}^{-1}$ and $L_{0.2-10}=$ $(3.3 \pm 1.0) \times 10^{38} \mathrm{erg} \mathrm{s}^{-1}$, respectively (assuming $9.6 \mathrm{Mpc}$; P08). Rebinning the data into two epochs (2008 March 5 to 2008 March 31 and 2008 April 4 to 2008 April 26) with similar exposure times ( $24.3 \mathrm{ks}$ and $27 \mathrm{ks}$, respectively) shows the X-ray source faded by $\approx 4$ from $L_{0.2-10}=(6.0 \pm 1.9) \times 10^{38} \mathrm{erg} \mathrm{s}^{-1}$ to $(1.4 \pm 0.9) \times 10^{38} \mathrm{erg} \mathrm{s}^{-1}$ during the observations.

We further analyzed archival pre-SN ACIS data obtained on 2000 November 3, 2004 July 29, and 2004 November 20 to characterize the X-ray contamination of the SN with the nearby X-ray source. No X-ray source is visible at the position of the SN (Figure 1, right), but the nearby X-ray source is clearly detected, with an average luminosity of $L_{0.2-10}=(5.9 \pm 0.4) \times 10^{38} \mathrm{erg} \mathrm{s}^{-1}$. A comparison of the X-ray luminosity for each of the three epochs shows the source is not variable. We therefore extracted the counts from the position of the SN from the XRT data using a larger aperture corresponding to the $100 \%$ encircled energy radius of the XRT PSF, which contains the nearby X-ray source, and subtracted the luminosity of the Chandra source. Within the errors, the same residual Xray luminosity for the source at the position of the $\mathrm{SN}$ is obtained when compared to the above analysis using a smaller aperture.

Radio observations were made with the VLA ${ }^{15}$ at $1.3,3.6$, 6.0 , and $20 \mathrm{~cm}$ in continuum mode. Initial data reduction and analysis of the radio data was similar to the methods used for SNe 1993J and 2001gd (Weiler et al. 2007; Stockdale et al. 2007).

SN 2008ax was observed with the HET on 2008 March 19 and 2008 March 28, for 600 and $1200 \mathrm{~s}$, respectively. The Low Resolution Spectrograph (LRS; Hill et al. 1998) was used with a $2^{\prime \prime}$ slit $(R \sim 300 ; \Delta \lambda \sim 4500-10,000 \AA)$. Standard $\operatorname{IRAF}^{16}$ reduction techniques of bias subtraction, flat fielding, and wavelength calibration were used. Relative flux calibration was performed using several flux standards (BD262606, HD84937, and HZ44; Fukugita et al. 1996; Massey \& Gronwall 1990) observed during 2008 March. The spectra are displayed in Figure 3.

\section{RESULTS}

Observations of SN 2008ax began $\sim 1.5$ days after explosion and continued until day 54. The SN was detected in all UVOT color filters, but after template subtraction, fewer detections remained in the $u v m 2$ and $u v w 2$ filters. Assuming a SN 1993J-like UV spectrum, this is expected since the UV flux is intrinsically fainter due to line blanketing, and $E(B-V)=0.3$ (P08) therefore the UV flux is more suppressed (see Table 2).

\footnotetext{
15 The VLA of the National Radio Astronomy Observatory is operated by Associated Universities, Inc. under a cooperative agreement with the National Science Foundation.

${ }^{16}$ IRAF is distributed by the National Optical Astronomy Observatory, which is operated by the Association of Universities for Research in Astronomy, Inc., under cooperative agreement with the National Science Foundation.
} 


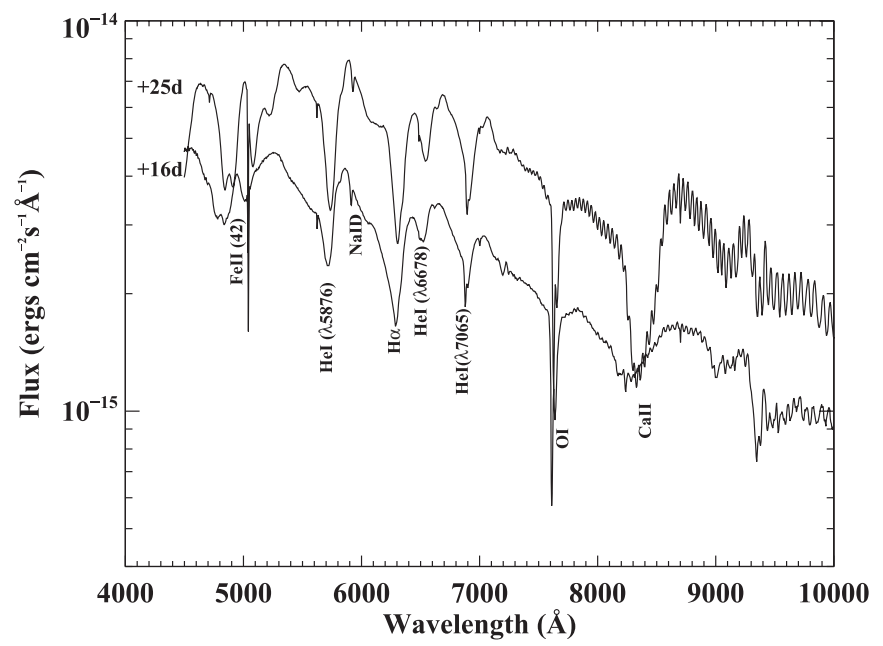

Figure 3. HET LRS spectra of SN 2008ax taken 16 and 25 days after explosion. Prominent lines specified in Jeffery et al. (1994) and P08 are indicated. The spectra have not been corrected for redshift or extinction. The $+25 \mathrm{~d}$ spectrum is shifted down by a constant factor of $50 \%$ for clarity.

The general trend for the light curves is that the UV bands tend to rise more rapidly, peak earlier, and decay more rapidly than the optical bands (see Table 1). This trend is also seen in a sample of UV/optical light curves of Type II SNe (Brown et al. 2009). The $v$ and $b$ SN 2008ax light-curve shapes are consistent with those of P08. A small shoulder in the $b$ is also seen in the first observation.

Also evident is an initial fading in the $u$ and $u v w 1$ light curves followed by a rise reminiscent of the dip seen in SNe 1987A (Type II; Hamuy et al. 1988), 1993J (IIb; Schmidt et al. 1993), 1999ex (Ibc; Stritzinger et al. 2002, hereafter S02), 2008D (Ibc; Soderberg et al. 2008), and 2006aj (Ic; Campana et al. 2006). The same shoulder in the $B$ and the upturn in the $U$ seen in $\mathrm{SN} 1999$ ex on day 4 after the explosion (S02) is also seen in the UVOT $b$ and $u$ data for SN 2008ax on approximately day 4. Additionally, a UV upturn is present which is the first time this has been seen in a Type IIb SN and only the third time in any $\mathrm{SNe}$ (cf., SNe 1987A \& 2006aj; Pun et al. 1995; Campana et al. 2006). This dip in the light curve is attributed to adiabatic cooling after the initial shock breakout (S02), and reinforces the idea that the progenitor is the result of a core collapse of a massive star (S02). Using Hubble Space Telescope (HST) data, Crockett et al. (2008) further constrained that the SN 2008ax progenitor was either a single massive star that lost most of its $\mathrm{H}$-envelope prior to exploding or an interacting binary in a low-mass cluster.

Using the method described by Richardson et al. (2006), we performed a Monte Carlo simulation with $2.5 \times 10^{5}$ realizations of the SN 2008ax $v$-band light curve ${ }^{17}$ by varying three model parameters: kinetic energy $\left(E_{k}\right)$, ejected mass $\left(M_{\mathrm{ej}}\right)$, and ejected nickel mass $\left(M_{\mathrm{Ni}}\right)$. The best $\chi^{2}$ fit resulted in $E_{k}=0.5 \times 10^{51} \mathrm{erg}, M_{\mathrm{ej}}=2.9 M_{\odot}$, and $M_{\mathrm{Ni}}=0.06 M_{\odot}$. The resultant parameters are consistent with values calculated by Richardson et al. (2006) for other Type IIb SNe, except that the SN 2008ax value for $M_{\mathrm{ej}}$ is $\sim 3 \times$ higher.

A $(u-b)$ and $(b-v)$ color plot (Figure 2, lower left) tracks temperature variations in the photosphere (Stritzinger

\footnotetext{
17 The Jeffery (1999) portion of the model used by Richardson et al. (2006) was excluded from our simulation since no late-time data are included in our sample. The Arnett (1982) portion of the model alone fits the light curves reasonably well.
}

et al. 2009, hereafter S09). Initially, the blue to red jump $(\Delta(u-b)=1.0)$ is due to the adiabatic cooling discussed previously. This is followed by a transition back to the blue as the photospheric temperature increases and the light curves reach maximum. As the temperature decreases, the color curves transition back to the red and begin to level off. Of note is that the $u$ and $u v w 1$ light curves stop declining in brightness at $\sim 25$ days after maximum. This corresponds with the color evolution to the blue in the $(u-b)$ at the same time. This blue excess was also seen in SNe 2007Y and 2008aq, and was attributed to a change in the opacity of the ejecta, or shock heating produced by interaction of high-velocity SN ejecta with circumstellar material (CSM; S09).

The lack of an X-ray source in pre-SN Chandra images and the detection of a fading X-ray source in Swift XRT data confirms that X-ray emission is detected from SN 2008ax. These $\mathrm{X}$-rays likely arise from interaction of the outgoing SN shock with substantial amounts of CSM. If the CSM was deposited by a strong stellar wind prior to outburst, as expected for the massive progenitor of a SN IIb, the X-ray luminosity can be used to estimate the mass-loss rate. Following the discussion in Immler et al. (2007), we infer a mass-loss rate of the progenitor of $\dot{M}=(9 \pm 3) \times 10^{-6} M_{\odot} \mathrm{yr}^{-1}\left(v_{\mathrm{w}} / 10 \mathrm{~km} \mathrm{~s}^{-1}\right)$ for an assumed speed of the outgoing shock of $v_{\mathrm{s}}=10,000 \mathrm{~km} \mathrm{~s}^{-1}$ and scaled for a stellar wind speed of $v_{\mathrm{w}}=10 \mathrm{~km} \mathrm{~s}^{-1}$. Only two other SN IIb have been detected in X-rays over the past four decades: SNe 1993J (Chandra et al. 2009) and 2001gd (Pérez-Torres et al. 2005). In contrast, SN 2008ax is only a weak X-ray emitter, 1 and 2 orders of magnitude fainter than SNe 2001gd and 1993J, respectively. Subsequently, the inferred mass-loss rate for the progenitor of SN 2008ax is significantly below that of SN 1993J $\left(10^{-5}-10^{-4} M_{\odot} \mathrm{yr}^{-1}\right.$; Immler et al. 2001) and more characteristic of SNe IIP $\left(10^{-6}-10^{-5} M_{\odot} \mathrm{yr}^{-1}\right.$; see Chevalier et al. 2006; Immler et al. 2007). This wide range of massloss rates for $\mathrm{SNe}$ IIb could be caused by a diversity of binary progenitor systems. ${ }^{18}$

The general nature of the SN 2008ax radio light curve (Figure 2, right) is very typical of core-collapse SNe (CCSNe; Martí-Vidal et al. 2009; Weiler et al. 2002). However, the time evolution of the radio emission is somewhat unusual for a Type IIb radio SN when compared to SNe 1993J, 2001gd, and 2001ig (Weiler et al. 2007; Stockdale et al. 2007; Ryder et al. 2004, 2006). SN 2008ax reached its $6 \mathrm{~cm}$ peak radio luminosity in 14 days, while SN 1993J took 133 days after explosion to achieve peak (Weiler et al. 2007). The exact time to reach this point is uncertain for both SNe 2001gd and 2001ig, but the parameterized models outlined in Weiler et al. (2002) indicate the peak occurred at $\sim 100$ days (Stockdale et al. 2007; Ryder et al. 2004). In contrast, the first event associated with a Type Ic SN and a gamma-ray burst, SN 1998bw/GRB 980425, reached its peak $6 \mathrm{~cm}$ radio luminosity in 10 days (Weiler et al. 2001).

A typical radio-derived mass-loss rate for the earlier Type IIb SNe is $10^{-5} M_{\odot} \mathrm{yr}^{-1}$ (Weiler et al. 2002). The exact radioderived mass-loss rate for SN 2008ax is difficult to establish as the exact nature of the $\mathrm{SN}$ progenitor is ambiguous (Crockett et al. 2008). Following the discussion in Weiler et al. (2002), we infer a range of possible mass-loss rate of the progenitor of $\dot{M}=(1-6) \times 10^{-6} M_{\odot} \mathrm{yr}^{-1}\left(v_{\mathrm{w}} / 10 \mathrm{~km} \mathrm{~s}^{-1}\right)$ for an assumed speed of the outgoing shock of $v_{\mathrm{s}}=10,000 \mathrm{~km} \mathrm{~s}^{-1}$ and scaled for a stellar wind speed of $v_{\mathrm{w}}=10 \mathrm{~km} \mathrm{~s}^{-1}$. The ambiguity

\footnotetext{
18 Note: SN 1993J does not show signs in its radio evolution of having been in a binary system (Weiler et al. 2007), although other wave bands indicate otherwise (Van Dyk 2004).
} 
of the nature of the progenitor makes the wind speed of the progenitor-established CSM very uncertain.

Furthermore, the radio evolution of SN 2008ax appears to show short timescale modulations of 5-10 days (see Figure 2, right). This may be similar to the evolution of SN 2001ig whose progenitor was attributed to an interacting-binary system or the evolution of SN 1998bw whose progenitor was a very massive star (Ryder et al. 2004, 2006; Weiler et al. 2001). A deeper analysis of the radio evolution of SN 2008ax is ongoing.

Spectroscopic observations of SN 2008ax occurred 16 and 25 days after explosion. The HET spectra (Figure 3) are very similar-with prominent $\mathrm{H} \alpha$ and $\mathrm{He}$ I lines - to SNe 1993J (Jeffery et al. 1994) and 2008ax (P08) spectra taken 18 and 28 days postexplosion, respectively. P08 showed at 56 days that Ca II was also present in SN 2008ax, but presented no data redward of $\sim 8000 \AA$ previous to this time. HET spectra reveal that the Ca II absorption line was significant at 16 days $(\sim 3$ days before $b$-peak) and was a factor of $\sim 3$ larger 9 days later. This behavior is reminiscent of SNe 2005bf (Ibc; Folatelli et al. 2006) and 2007Y (Ib; S09). Both SNe had very early strong Ca II absorption lines, followed by a decrease in strength, and then a rapid increase in strength. Assuming the early-time Ca II absorption in SN 2008ax was also strong and then weakened, the HET spectra indicate around peak and after peak that $\mathrm{Ca}$ II is caused by a photospheric, rather than a high-velocity component above the photosphere (S09; Parrent et al. 2007). The behavior of the blueward components of the HET spectra, particularly the strengthening of the He I lines, are consistent with the P08 results.

\section{CONCLUSION}

The $u$ and $u v w 1$ light curves of SN 2008ax show a rapid initial decline to a minimum approximately 4 days after shock breakout. The SN then brightens to maximum light in the $u$ band at 17.2 \pm 0.4 days after shock breakout. This behavior is seen in other CCSNe and is predicted in numerical models of CCSNe with stripped atmospheres (Shigeyama et al. 1990, 1994). However, it is not seen in most CCSNe light curves. This may be due to the paucity of detailed early-time observations for most $\mathrm{SNe}$, or indicate a physical difference in the progenitors. The dip is attributed to rapid adiabatic cooling of the photosphere, which is accelerated by the shock passage. The timescale of adiabatic cooling depends on the volume of the photospheric shell, and thus the radius of the progenitor. Modeling of CCSNe with stripped atmospheres suggests that the adiabatic cooling acts on timescales of several days, which is in agreement with the UV minimum seen in SN 2008ax.

Shigeyama et al. (1990) produced models of exploding helium stars, which are believed to produce $\mathrm{SNe}$ IIb. The model assumes that the progenitor is a stripped star in a binary system. From the X-ray and radio mass-loss rates coupled with the short time evolution of the radio emission, the SN 2008ax progenitor appears to have been in an interacting binary, consistent with the work of Crockett et al. (2008). The observed time of maximum light and shape of the later SN 2008ax light curve are consistent with an unmixed progenitor model which produces early UV dips lasting longer than those with mixing in the progenitor. Dips in mixed models tend to have minima at $\lesssim 5$ days while those in unmixed models tend to have minima at $\approx 5-10$ days. The time of the SN 2008ax dip is consistent with a mixed progenitor; however, the time is uncertain due to the lack of very early-time data. It is therefore possible that the true minimum occurred later than $\approx 4$ days, as is predicted for unmixed models. Further evidence for an unmixed progenitor comes from the strength of the dip. The observed dip is $\approx 4$ mag consistent with an unmixed model. These three lines of evidence suggest that the progenitor of SN 2008ax was either unmixed, or very lightly mixed.

Shigeyama et al. (1990) find that helium stars with less mixing tend to be more massive than those that undergo extensive mixing. If the progenitor of SN 2008ax is unmixed then it may be on the more massive side of the progenitor mass distribution. This supports our result that the mass-loss rate from SN 2008ax is lower than for a typical Type IIb SNe.

The onset and strength of the adiabatic cooling dip increases as the progenitor mass increases, so more massive progenitors have more prominent adiabatic cooling following the shock breakout. This suggests that the lack of an observed cooling dip for many SNe IIb may be due to less massive progenitors. This, combined with the paucity of high-cadence early-time observations, may explain why the cooling dip is not seen in many SNe IIb.

We thank Alicia Soderberg for suggestions regarding this work, which is sponsored at PSU by NASA contract NAS500136 and at Marquette by NASA award NNX09AC90G. The HET of UT-Austin, PSU, Stanford, Ludwig-MaximiliansUniversität München, and Georg-August-Universität Göttingen, is named in honor of William Hobby and Robert Eberly.

\section{REFERENCES}

Arnett, W. D. 1982, ApJ, 253, 785

Brown, P. J., et al. 2009, AJ, 137, 4517

Burrows, D. N., et al. 2005, Space Sci. Rev., 120, 165

Campana, S., et al. 2006, Nature, 442, 1008

Cardelli, J. A., Clayton, G. C., \& Mathis, J. S. 1989, ApJ, 345, 245

Chandra, P., Dwarkadas, V. V., Ray, A., Immler, S., \& Pooley, D. 2009, ApJ, 699, 388

Chevalier, R. A., Fransson, C., \& Nymark, T. K. 2006, ApJ, 641, 1029

Crockett, R. M., et al. 2008, MNRAS, 391, L5

de Boer, K. S., Pascual, P. R., Wamsteker, W., Sonneborn, G., Fransson, C., Bomans, D. J., \& Kirshner, R. P. 1993, A\&A, 280, L15

Dickey, J. M., \& Lockman, F. J. 1990, ARA\&A, 28, 215

Filippenko, A. V. 1988, AJ, 96, 1941

Folatelli, G., et al. 2006, ApJ, 641, 1039

Fordham, J. L. A., Moorhead, C. F., \& Galbraith, R. F. 2000, MNRAS, 312, 83

Fransson, C., Lundqvist, P., \& Chevalier, R. A. 1996, ApJ, 461, 993

Fukugita, M., Ichikawa, T., Gunn, J. E., Doi, M., Shimasaku, K., \& Schneider, D. P. 1996, AJ, 111, 1748

Gehrels, N., et al. 2004, ApJ, 611, 1005

Hamuy, M., Suntzeff, N. B., González, R., \& Martin, G. 1988, AJ, 95, 63

Hill, G. J., Nicklas, H. E., MacQueen, P. J., Tejada, C., Cobos Duenas, F. J., \& Mitsch, W. 1998, Proc. SPIE, 3355, 375

Immler, S., Aschenbach, B., \& Wang, Q. D. 2001, ApJ, 561, 107

Immler, S., et al. 2007, ApJ, 664, 435

Jeffery, D. J. 1999, arXiv:astro-ph/9907015

Jeffery, D. J., et al. 1994, ApJ, 421, 27

Martí-Vidal, I., et al. 2009, A\&A, 499, 649

Massey, P., \& Gronwall, C. 1990, ApJ, 358, 344

Mostardi, R., Li, W., \& Filippenko, A. V. 2008, Central Bureau Electronic Telegrams, 1280, 1

Nomoto, K., Suzuki, T., Shigeyama, T., Kumagai, S., Yamaoka, H., \& Saio, H. 1993, Nature, 364, 507

Parrent, J., et al. 2004, PASP, 119, 135

Pastorello, A., et al. 2008, MNRAS, 389, 955 (P08)

Pei, Y. C. 1992, ApJ, 395, 130

Pérez-Torres, M. A., et al. 2005, MNRAS, 360, 1055

Poole, T. S., et al. 2008, MNRAS, 383, 627

Pun, C. S. J., et al. 1995, ApJS, 99, 223

Qiu, Y., Li, W., Qiao, Q., \& Hu, J. 1999, AJ, 117, 736

Ramsey, L. W., et al. 1998, Proc. SPIE, 3352, 34

Richardson, D., Branch, D., \& Baron, E. 2006, AJ, 131, 2233

Roming, P. W. A., et al. 2005, Space Sci. Rev., 120, 95 
Ryder, S. D., et al. 2004, MNRAS, 349, 1093

Ryder, S. D., et al. 2006, MNRAS, 369, L32

Schmidt, B. P., et al. 1993, Nature, 364, 600

Shigeyama, T., Nomoto, K., Tsujimoto, T., \& Hashimoto, M. 1990, ApJ, 361, L23

Shigeyama, T., Suzuki, T., Kumagal, S., Nomoto, K., Saio, H., \& Yamaoka, H. 1994, ApJ, 420, 341

Soderberg, A. M., et al. 2008, Nature, 453, 469
Stockdale, C. J., et al. 2007, ApJ, 671, 689

Stritzinger, M., et al. 2002, AJ, 124, 2100 (S02)

Stritzinger, M., et al. 2009, ApJ, 696, 713 (S09)

Van Dyk, S. D. 2004, New Astron. Rev., 48, 749

Weiler, K. W., Panagia, N., \& Montes, M. J. 2001, ApJ, 562, 670

Weiler, K. W., et al. 2002, ARA\&A, 40, 387

Weiler, K. W., et al. 2007, ApJ, 671, 1959

Woosley, S. E., Pinto, P. A., Martin, P. G., \& Weaver, T. A. 1987, ApJ, 318, 664 